\title{
IDENTIFICATION AND QUANTIFICATION OF FACTORS AFFECTING REUSABILITY OF OPEN SOURCE SOFTWARE IN REUSE-INTENSIVE SOFTWARE DEVELOPMENT
}

\author{
Fazal-e-Amin ${ }^{1}$, Aized Amin Soofi ${ }^{2}$ \\ ${ }^{1}$ Department of Software Engineering \\ College of Computer and Information Sciences, \\ King Saud University, Riyadh, Saudi Arabia \\ ${ }^{2}$ Department of Computer Science, Allama Iqbal Open University, Islamabad, Pakistan \\ e-mail: famin@ksu.edu.sa, aizedamin@yahoo.com
}

\begin{abstract}
Open Source Software (OSS) is one of the emerging areas in software engineering. Reuse of OSS is employed in reuse-intensive software development such as Component Based Software Development and Software Product Lines. OSS is gaining the interest of the software development community due to its enormous benefits. The context of this study is the identification and quantification of factors affecting reusability of OSS in reuse-intensive software development. The use of OSS in the systematic reuse of software, such as in Software Product Lines (SPLs) is a new phenomenon. Therefore, the aim of this study is to identify and quantify the factors affecting the reusability of OSS in reuse-intensive software development, especially for SPLs. In this study, a mixed methods based approach is employed to identify the factors affecting the reusability of OSS. Interviews are conducted with experts in this field as the qualitative part, followed by a survey, experiments and a statistical analysis. The factors identified during the interviews are ranked by software engineers in a survey. Experiment is conducted to assess the reusability of open source packages. The factors are validated by conducting a statistical analysis of the results of the experiments. A set of nine factors were identified as a result of the qualitative study. A model was formed on the basis of the findings of interviews and a survey. It includes five factors. These were statistically analyzed by applying the model to 77 open source packages. A set of nine factors were identified as affecting reusability of OSS in a reuse-intensive development environment. Five of them were validated at the code level. The statistical results show a positive correlation between reusability and the identified factors.
\end{abstract}

Keywords: Open source software; software reusability; mixed method

\section{INTRODUCTION}

OSS is gaining the interest of the software engineering community due to its numerous benefits. These benefits fall into different dimensions. One dimension is 'financial benefits', which includes the reduction in maintenance cost (Niemi, Tuisku, Hameri, \& Curtin, 2009) and escape from vendor lock-in (Niemi, et al., 2009; Stafford, 2006). The technical benefits of OSS include having a large number of developers and testers 
(Kenwood, 2001; Krishnamurthy, 2003) and the lessening of maintenance risk (F. v. d. Linden, Lundell, \& Marttiin, 2009). Other benefits include user support from the community (Krishnamurthy, 2003), encouraging innovation (Howe, 2000; Wheeler, 2005) and increased collaboration (P. J. Ågerfalk, Deverell, Fitzgerald, \& Morgan, 2005), which are multifaceted advantages of OSS. These may be seen from the social aspect or from the financial aspect. The things which contribute to the popularity of OSS may also include the availability of increased bandwidth, search facilities, and tools such as Code Conjurer (Hummel, Janjic, \& Atkinson, 2008). The growth of the Internet is also one of the things that has had a huge impact on the way software is developed, marketed, and supported (Wasserman, 2011). The use of OSS in CBSD is already a norm in the industry (Sommerville, 2007). Recently the researchers have envisioned the use of OSS in SPL development (P. Agerfalk et al., 2006; Ahmed, Capretz, \& Babar, 2008). So, OSS is entering into a new arena. Currently limited knowledge is available in this research area. There is a need to explore the use of OSS in reuse-intensive software development especially in SPL.

The disciplines of OSS, CBSD, and SPL share a common theme, namely 'reusability'. A broader definition of reusability describes it as the "reapplication of various kinds of knowledge about one system to another system in order to reduce the effort of developing and maintaining that system" (Samadi, Almaeh, Wolfe, Olding, \& Isaac, 2004). OSS is developed to be reused and contributed to by numerous software engineers.

The reuse-intensive software development methodologies such as CBSD and SPL reuse software artefacts to develop new products. In CBSD, software components are composed to develop software systems. In SPL software assets are 'developed to reuse' and 'reused for development' (F. Linden, Schmid, \& Rommes, 2007). The concept of reusability is the central tenet in these areas. So, in the context of OSS based development of CBSD /SPL it is important to assess the reusability of an asset. Reusability assessment is recognized as a research area in software engineering (Frakes \& Kyo, 2005).

In (Frakes \& Kyo, 2005), it is pointed out that reuse assessment is necessary to make software reuse a scientific and engineering approach. Several attempts such as (Boxall \& Araban, 2004; Gui \& Scott, 2007; Gui \& Scott, 2008; Sharma, Grover, \& Kumar, 2009; Yoonjung, Sungwook, Houp, Jingoo, \& SunHee, 2008) have been made to assess the reusability of software assets. The collection and synthesis of these works needs time. It will provide insight into the current state of the art and help to identify the short comings which will pave the way forward. Following this the factors affecting reusability of software will be identified. A reusability assessment model can be formed to assess the reusability of a code asset. The importance of understanding and analysis of code cannot be neglected. As the code base is increasing, the importance of its analysis and manipulation is also increased (Harman, 2010).

As stated earlier, the nature of this study is exploratory. A review of current approaches reveals that none of the approaches proposed considered the emerging situation arising due to the combination of OSS and SPLs (Fazal-e-Amin, Ahmad Kamil Mahmood, \& Alan Oxley, 2011d). Reusability can be viewed as usability from the perspective of the developer (Burgin, Lee, \& Debnath, 2004). Usability is a subjective phenomenon. An interview can be used to have an insight into the opinions of the informants. In this study these concerns are undertaken and the interview is used as a 
tool to explore the phenomenon of reusability in reuse-intensive software development and OSS.

Software reuse based development has become a standard in business and commercial software development (Sommerville, 2007). Software reuse is commonly employed in two ways, first by using component libraries, as in CBSD, and second in a systematic way as in software product line development. This focuses on reuse intense development. Software artefacts are developed, from existing artefacts, with the intention of being reused. The product line development concept revolves around the terms 'commonality' (the requirements which are common to family members) and 'variability' (the distinguishing requirements).

The three generic activities for using a component in CBSD are identification, selection and adaptation of components (where this is necessary). In the past, the decision to be made was to develop a component or to buy one. Now, with the emergence of OSS, there is a third option (Höst, Oručević-Alagić, \& Runeson, 2011). During the selection of components, different criteria are used. These may include legal aspects, such as license type or maintenance support for the component. In the context of this paper, a particular criterion, the reusability of a component, is assessed to facilitate the decision process.

\section{RESEARCH METHODOLOGY}

In this study, a mixed method approach is used to achieve the objectives. Mixed method research is emerging as a third option for researchers in addition to qualitative and quantitative research (Leech \& Onwuegbuzie, 2009). Mixed method studies combine the qualitative and quantitative approaches. (Tashakkori \& Teddlie, 1998) comments on mixed method studies: "These are studies that are the products of pragmatist paradigm and that combine the qualitative and quantitative approaches within different phases of the research process".

Software engineering is a multidisciplinary field. It deals with social and technological issues. A software engineering activity is not only based on the processes and tools, but it also depends on the social and cognitive process around it (Easterbrook, Singer, Storey, \& Damian, 2008). Therefore, study of human activities is necessary to understand a problem and its solution in the software engineering domain. The aforementioned importance of human activities in the software engineering field compels us to use the research methods of fields related to the study of human behavior.

In the software engineering field, opinions exist that suggest there should be a combination of both qualitative and quantitative research methods in software engineering research (Lazaro \& Marcos, 2006). The authors also state that using a combination of qualitative and quantitative methods may be beneficial in that it provides information from a number of perspectives. The combination of qualitative and quantitative approaches in a single study is referred to as a mixed method study.

An example of mixed method studies in software engineering can be found in (Wood, Daly, Miller, \& Roper, 1999), where an investigation of object orientation is made using a survey questionnaire, a structured interview and controlled laboratory experiments. 


\section{Research Questions}

Following research questions are answered in this study:

RQ1 - What are the factors affecting reusability of open source software in reuseintensive software development?

RQ2 - How to measure the factors affecting reusability?

RQ3 - What is the significance and nature of the relationship between reusability and its identified factors?

\section{Research Methods}

The research methods used in this study are presented in the next sections.

\section{Interview}

The interview is a means of collecting primary data; it is a conversation between two persons, one of whom is a researcher. Interviews can be used for data collection where the nature of the study is exploratory. Interviews are helpful when the data to be gathered is about a person's knowledge, preferences, attitude or values (Gray, 2009). Several types of interviews are reported in the literature (Punch, 2009). In this study, the semi-structured type of interview is used.

Semi-structured interviews make use of both open-ended and specific questions. This combination allows the researcher to explore the issues and to collect expected information using specific questions while open-ended questions provide unforeseen information.

\section{Respondents' Profiles}

The research issues investigated in this study are of a specialized nature. Not everybody working in industry or academia is able to answer the questions. The respondents chosen for this study were based on their expertise. It should be noted that the respondents have up to date information regarding the research and industrial practices in this area.

The interviews were audio recorded and transcribed prior to performing the analysis. The first respondent is a software engineering researcher and developer. He has experience related to human computer interaction application development.

The second respondent is a researcher with a doctorate degree in software engineering in the area of software product lines. He is an author of many publications and book chapters, including those specifically targeting software product lines and related issues.

The third respondent is an expert in software reuse research, and has been authoring research papers on software reuse since the 1980s. He is actively participating in research activities and is currently the editor of a publication in software engineering published by a prestigious body. He is currently serving as principle software architect in a well-known organization.

The fourth respondent started his career as a software engineer and was promoted to software project manager during his career. He has managed projects in the 
domains of accounts, student information services, examination systems and a few others involving automating small industries and NGOs.

The fifth respondent has worked in the domains of micro finance systems, accounts systems, medical laboratory systems, visa systems, and billing systems.

\section{Qualitative Analysis Coding Process}

The content analysis approach is used in this study for the analysis of qualitative data. Content analysis is a scientific tool which helps to understand the phenomenon. The content analysis is a "research technique for making replicable and valid inferences from the text (or other meaningful matter) to the contexts of their use" (Krippendorff, 2004).

The analysis is conducted following the approach presented in (Elo \& Kyngäs, 2008) and (Hsieh \& Shannon, 2005). The analysis process is started by generating the transcriptions from the audio recorded interviews. These transcripts are read carefully to extract the open codes (Elo \& Kyngäs, 2008). The open coding process results in a list of codes. The open coding process is performed by using atlas.ti software. In this paper only one category i.e. factors affecting reusability is discussed, further details can be found in (Fazal-e-Amin, Ahmad Kamil Mahmood, \& Alan Oxley, 2011e).

\section{Survey Population and Sampling Technique}

The objective of software engineering research is to provide results which are useful for the software industry. The selection of a population for a survey is one of the critical decisions. In this study the target population consists of the individuals related to the software development in Malaysia.

In this research, sample is collected using convenience sampling. The driving force to make this decision is time and resource constraints. It's a non-probability sampling method. On contrary, the probability sampling method also requires the identification of every individual in the population which is quite difficult in this case.

There are two common strategies to minimize the sample biasness in convenience sampling. These include a clear description of sample collection process and participants, and to ensure that sample is reasonably representative without any bias (Gravetter \& Forzano, 2011). Both of these remedies are applied in this research. It is tried as much as possible during the data collection that data should be collected from the representative population. 


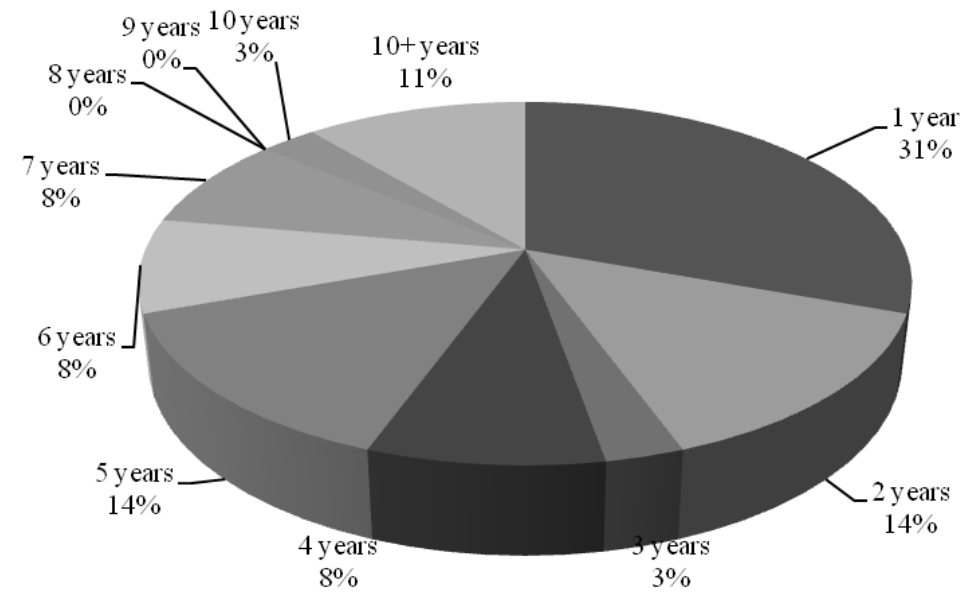

Figure 1. Experience of Survey population

Table 1 Survey Details

\begin{tabular}{|c|c|}
\hline \multicolumn{2}{|l|}{ Survey Details } \\
\hline Confidence level & $95 \%$ \\
\hline Population & $91410^{*}$ \\
\hline Sample size needed & 383 \\
\hline Population Accessed & $2707+$ \\
\hline Sample size collected & 396 \\
\hline Confidence interval & 4.91 \\
\hline Percentage & 50 \\
\hline \multicolumn{2}{|c|}{$\begin{array}{l}\text { Calculated using calculator } \\
\text { (www.surveysystem.com/scalc.htm) } \\
\text { *Population Estimation Source : (MSC Malaysia Supply-Demand } \\
\text { Study of ICT Industry) }\end{array}$} \\
\hline
\end{tabular}




\section{Statistical Analysis}

In this study, the Pearson correlation coefficient, $r$, was used as a numerical measure that assesses the strength of the linear relationship between two variables ('reusability' and 'factor').

In experiment, 77 packages were assessed for their reusability. The software was downloaded from multiple sources which include Sourceforge (www.sourceforge.net), Merobase (www.merobase.com), and FreashMeat (www.freshmeat.net).

\section{Factors Affecting Reusability}

The factors identified as affecting reusability are presented in this section. The factors and corresponding quotes are presented in Table 2. Further definitions of factors and metrics are presented in appendix.

Table 2. Identified factors and representative quotes

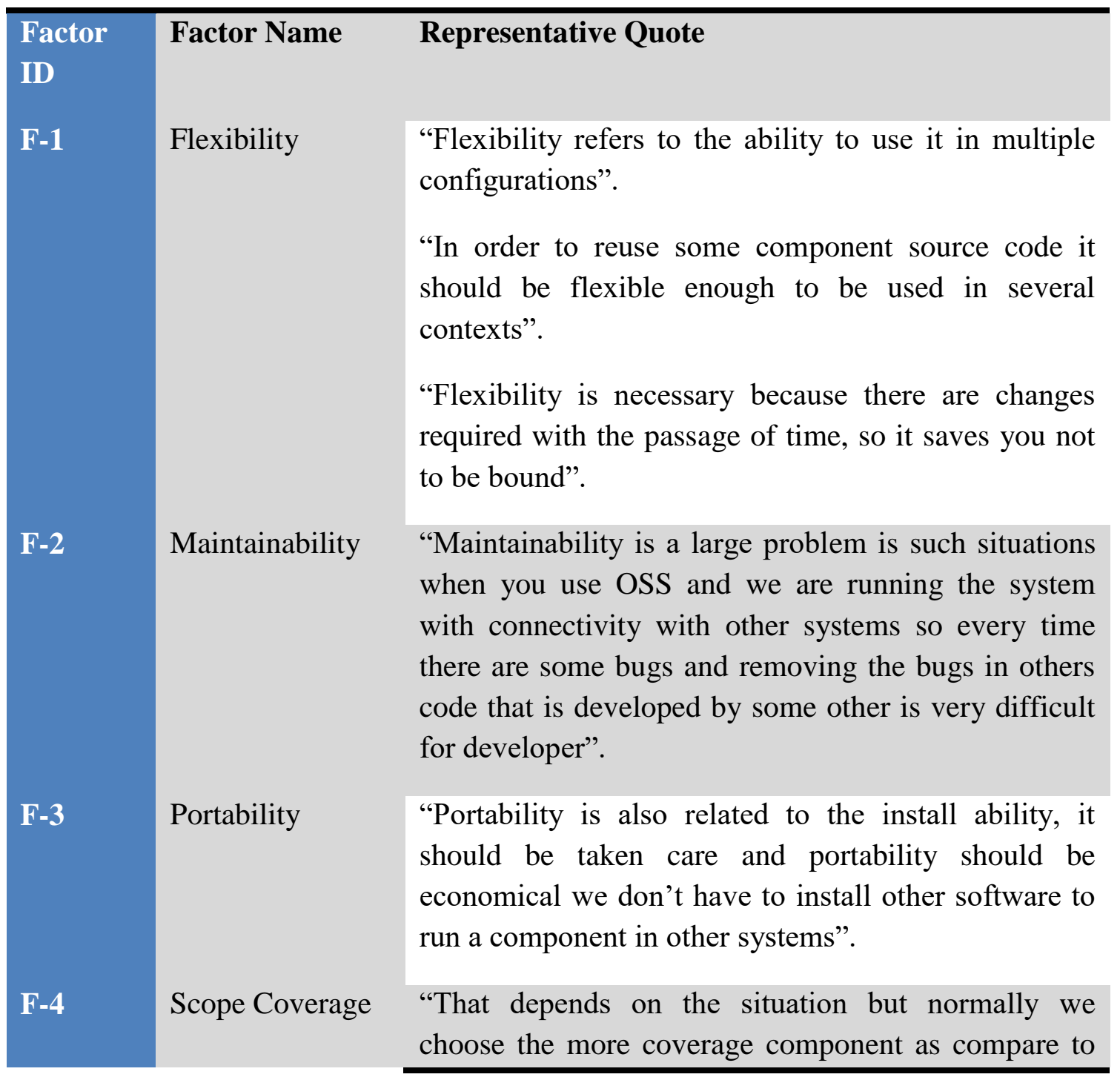




the less covered one".
".. it depends on the application if we want to extend
further our application then we will go for more
features".
"Stable meaning reasonably error free and it could be
used with confidence that there is no bug".
F-6
"If I don't understand it then I can't show that it is
reliable and prove it to myself then I am not going to
use it".
"Size can be managed but if it is not understandable
then it is difficult to reuse"
"Usage history also shows the maturity of the
component and how many people have used and made
changes to it".
"In many cases open source software is used by many
people many engineers, already proven its
usefulness".
"Variability is a two edge sword in other words there
are advantages and disadvantages".
"If there is lack of documentation then I mean it
creates hurdles to understand the code for any other
developer or the software engineer".
"If there is no proper documentation then others
cannot understand the software neither can change nor
modify it".

\section{Results of Survey}

The results of the survey are presented in the form of pie charts. The percentages of population selected a specific scales are presented in Figure 2 to 10.

The pie chart of attribute flexibility shows a concentrated data at scale four (agree) and five (strongly agree). It shows a consensus that flexibility is one of the attribute of reusability. 


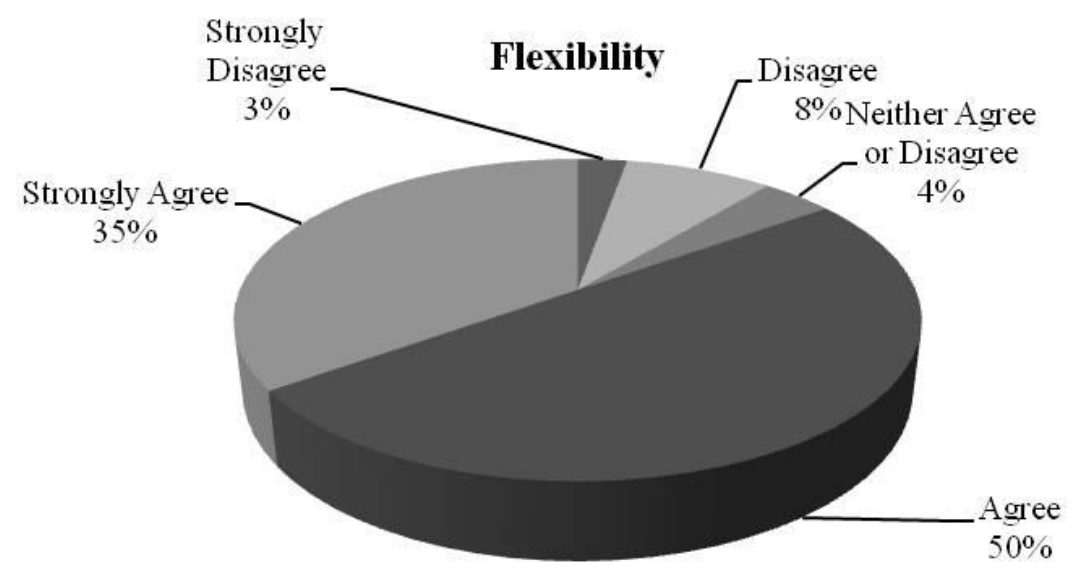

Figure 2. Frequency distribution of the scales assigned to flexibility

\begin{tabular}{lc}
\hline \multicolumn{1}{c}{ Scale } & Clexibility \\
\multicolumn{1}{c}{ Confidence Interval } \\
Strongly Disagree & $* 0 \%-7.69 \%$ \\
Disagree & $3.42 \%-13.24 \%$ \\
Neither Agree or Disagree & $* 0 \%-8.95 \%$ \\
Agree & $45.09 \%-54.91 \%$ \\
Strongly Agree & $29.94 \%-39.76 \%$
\end{tabular}

* The lower limit being below zero is rounded to zero, following the guidelines presented in (Beck, 2011).

The pie chart of scope coverage shows a less consensus of population that it is an attribute of reusability. It is visible by the percentage of population opted for the scale (disagree). 


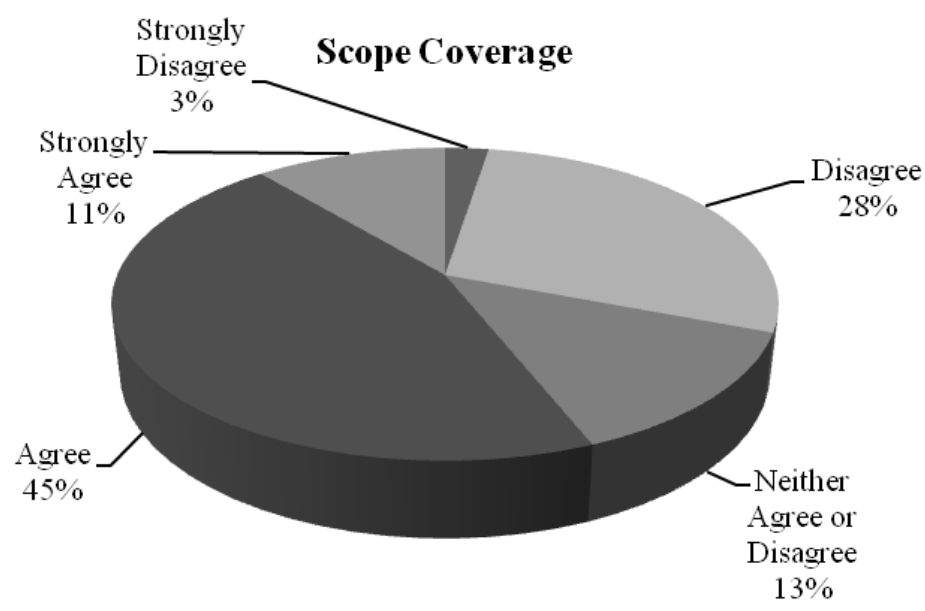

Figure 3. Frequency distribution of the scales assigned to scope coverage

\begin{tabular}{lc}
\hline \multicolumn{1}{c}{ Scale } & Scope Coverage \\
\multicolumn{1}{c}{ Confidence Interval } \\
Strongly Disagree & $* 0 \%-7.44 \%$ \\
Disagree & $23.37 \%-33.19 \%$ \\
Neither Agree or Disagree & $8.22 \%-18.04 \%$ \\
Agree & $40.04 \%-49.86 \%$ \\
Strongly Agree & $6.20 \%-16.02 \%$
\end{tabular}

* The lower limit being below zero is rounded to zero, following the guidelines presented in (Beck, 2011).

The pie chart of attribute portability shows a concentrated data at scale four (agree) and five (strongly agree). It shows a consensus that portability is one of the attribute of reusability. 


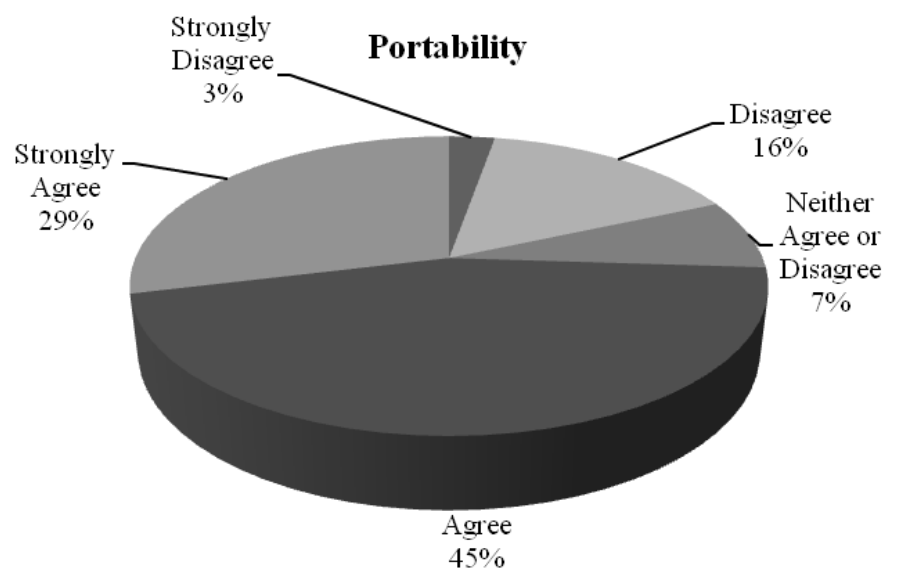

Figure 4. Frequency distribution of the scales assigned to portability

\begin{tabular}{lc}
\hline \multicolumn{1}{c}{ Scale } & Cortability \\
\multicolumn{1}{c}{ Confidence Interval } \\
Strongly Disagree & $* 0 \%-7.69 \%$ \\
Disagree & $10.75 \%-20.57 \%$ \\
Neither Agree or Disagree & $2.67 \%-12.49 \%$ \\
Agree & $40.29 \%-50.11 \%$ \\
Strongly Agree & $23.88 \%-33.70 \%$
\end{tabular}

* The lower limit being below zero is rounded to zero, following the guidelines presented in (Beck, 2011).

The pie chart of attribute maintainability shows a concentrated data at scale four (agree) and five (strongly agree). It shows a consensus that maintainability is one of the attribute of reusability. 


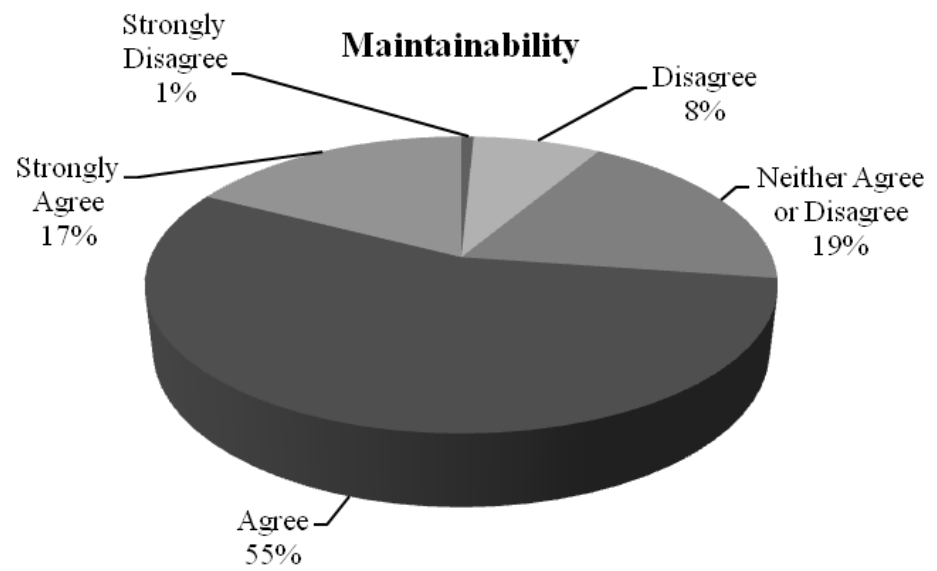

Figure 5. Frequency distribution of the scales assigned to maintainability

\begin{tabular}{lc}
\hline \multicolumn{1}{c}{ Scale } & Maintainability \\
\multicolumn{1}{c}{ Confidence Interval } \\
Strongly Disagree & $* 0 \%-5.67 \%$ \\
Disagree & $2.92 \%-12.74 \%$ \\
Neither Agree or Disagree & $13.78 \%-23.60 \%$ \\
Agree & $50.39 \%-60.21 \%$ \\
Strongly Agree & $12.51 \%-22.33 \%$
\end{tabular}

* The lower limit being below zero is rounded to zero, following the guidelines presented in (Beck, 2011).

The pie chart of attribute variability shows that $30 \%$ of the population opted for neither agree nor disagree. One of the possible reasons for this indecisiveness of population is lack of knowledge about the variability. 


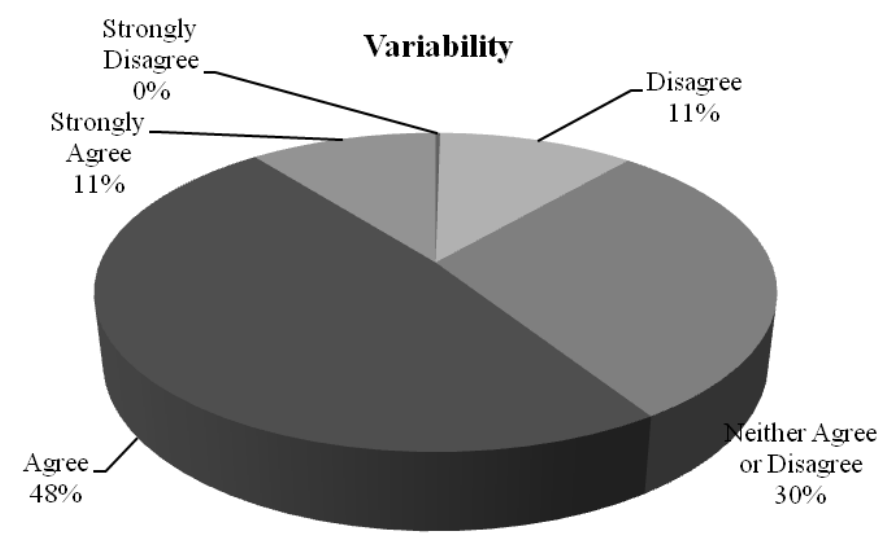

Figure 6. Frequency distribution of the scales assigned to variability

\begin{tabular}{lc}
\hline \multicolumn{1}{c}{ Scale } & Variability \\
Confidence Interval \\
Strongly Disagree & $* 0 \%-5.16 \%$ \\
Disagree & $6.20 \%-16.02 \%$ \\
Neither Agree or Disagree & $24.64 \%-34.46 \%$ \\
Agree & $43.57 \%-53.39 \%$ \\
Strongly Agree & $5.70 \%-15.52 \%$
\end{tabular}

* The lower limit being below zero is rounded to zero, following the guidelines presented in (Beck, 2011).

The pie chart of attribute understandability shows a concentrated data at scale four (agree) and five (strongly agree). It shows a consensus that understandability is one of the attribute of reusability. 


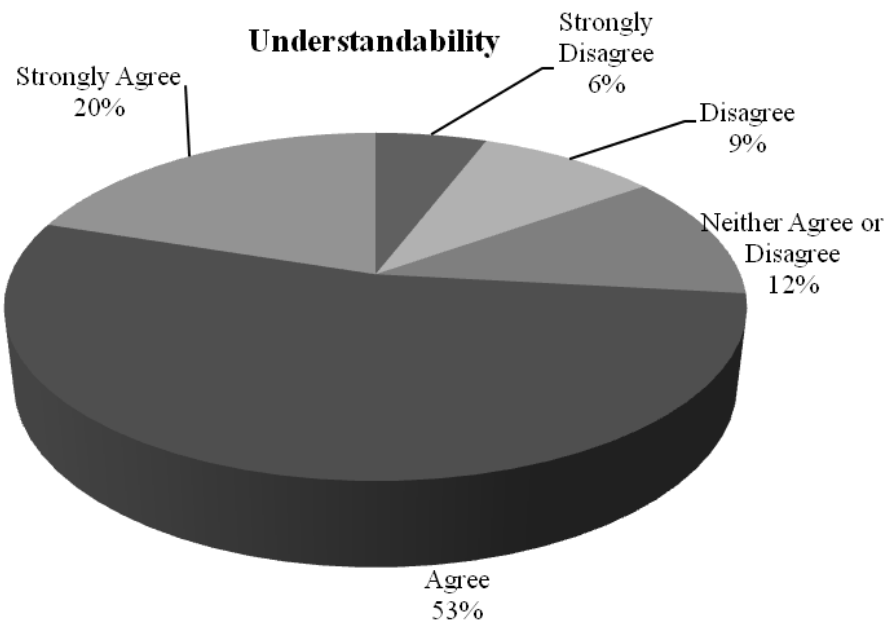

Figure 7. Frequency distribution of the scales assigned to understandability

\begin{tabular}{lc}
\hline \multicolumn{1}{c}{ Scale } & Confidence Interval \\
Strongly Disagree & $* 0 \%-10.72 \%$ \\
Disagree & $4.43 \%-\% 14.25$ \\
Neither Agree or Disagree & $6.71 \%-\% 16.53$ \\
Agree & $48.37 \%-\% 58.19$ \\
Strongly Agree & $15.04 \%-24.86 \%$
\end{tabular}

* The lower limit being below zero is rounded to zero, following the guidelines presented in (Beck, 2011).

The pie chart of documentation shows that a large number of populations opted for scales; agree $(31 \%)$ and strongly agree $(31 \%)$ population that documentation is an attribute of reusability. On the other hand, $24 \%$ of population opted for neither agree nor disagree and $14 \%$ of population is having a disagreement that documentation affects the reusability of software. 


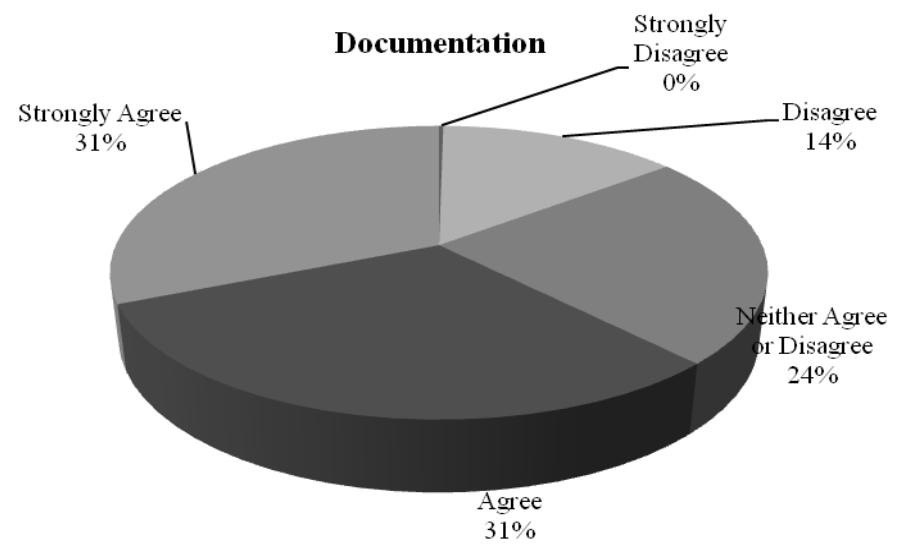

Figure 8. Frequency distribution of the scales assigned to documentation

\begin{tabular}{lc}
\hline \multicolumn{1}{c}{ Scale } & Documentation \\
\multicolumn{1}{c}{ Confidence Interval } \\
Strongly Disagree & $* 0 \%-5.16 \%$ \\
Disagree & $9.23 \%-19.05 \%$ \\
Neither Agree or Disagree & $18.57 \%-28.39 \%$ \\
Agree & $25.90 \%-35.72 \%$ \\
Strongly Agree & $26.40 \%-36.22 \%$
\end{tabular}

* The lower limit being below zero is rounded to zero, following the guidelines presented in (Beck, 2011).

The pie chart of attribute usage history shows a concentrated data at scale four (agree) and five (strongly agree). It shows a consensus that usage history is one of the attribute of reusability. 


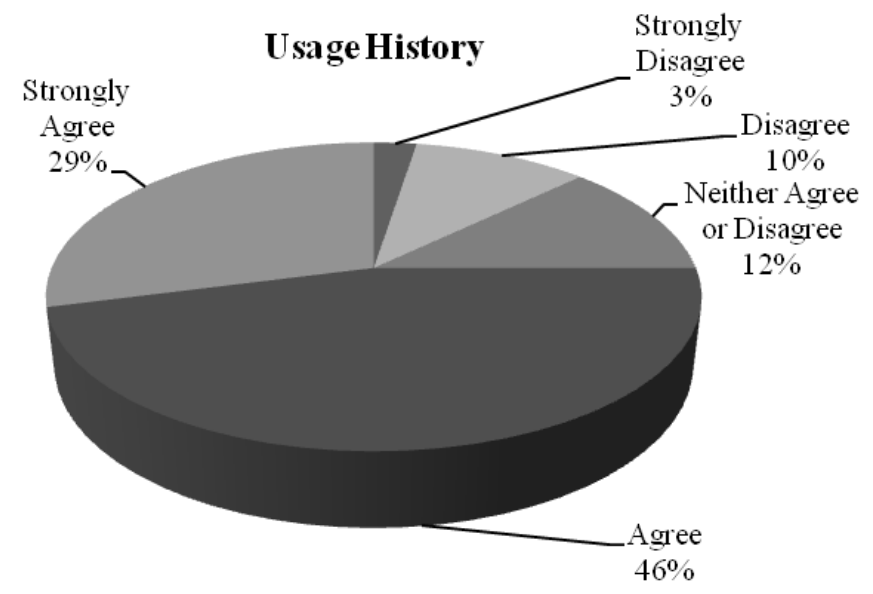

Figure 9. Frequency distribution of the scales assigned to usage history

\begin{tabular}{lc}
\hline \multicolumn{1}{c}{ Scale } & Usage History \\
\multicolumn{1}{c}{ Confidence Interval } \\
Strongly Disagree & $* 0 \%-7.44 \%$ \\
Disagree & $5.44 \%-15.26 \%$ \\
Neither Agree or Disagree & $7.21 \%-17.03 \%$ \\
Agree & $41.05 \%-50.87 \%$ \\
Strongly Agree & $24.13 \%-33.95 \%$
\end{tabular}

* The lower limit being below zero is rounded to zero, following the guidelines presented in (Beck, 2011).

The pie chart of reusability attribute stability shows concentrated data at scale four (agree) and five (strongly agree). It shows a consensus that stability is one of the attribute of reusability. 


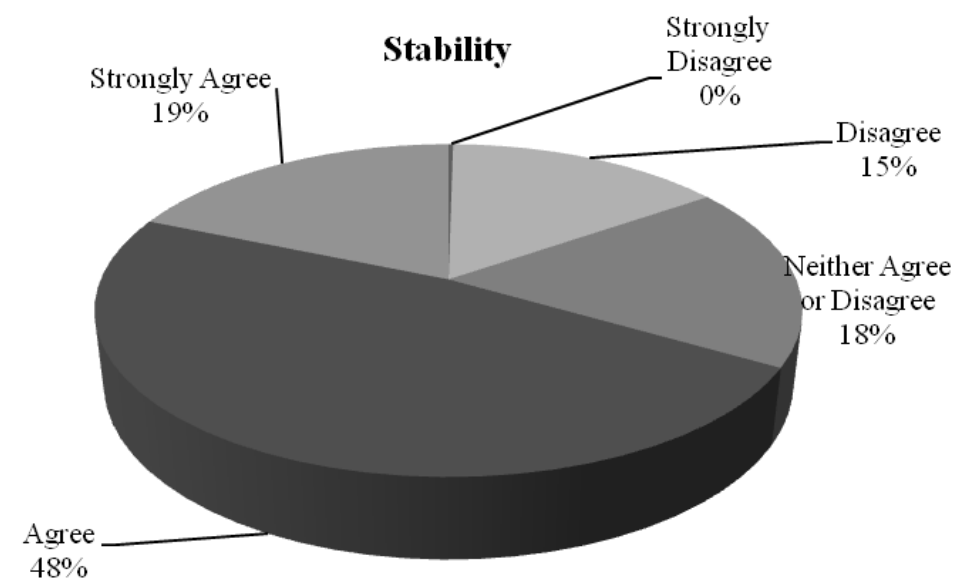

Figure 10. Frequency distribution of the scales assigned to stability

\section{Stability}

Scale

Strongly Disagree

Disagree

Neither Agree or Disagree

Agree

Strongly Agree

\section{Confidence Interval}

$$
* 0 \%-5.16 \%
$$

$10.24 \%-20.06 \%$

$13.02 \%-22.84 \%$

$43.07 \%-52.89 \%$

$13.78 \%-23.60 \%$

* The lower limit being below zero is rounded to zero, following the guidelines presented in (Beck, 2011).

\section{Factors}

\section{F-1 Flexibility}

It is defined as "the ease with which a system or component can be modified for use in applications or environments other than those for which it was specifically designed" (IEEE, 2010). In (Pohl, Böckle, \& Linden, 2005; Sant'anna, Garcia, Chavez, Lucena, \& von Staa, 2003; Sharma, et al., 2009) 'flexibility' is considered as a factor affecting the reusability of a component. In the context of an SPL, the flexibility characteristic is necessary for a core asset as it is intended to be reused in the development of other products.

Flexibility is related to reusability in two ways. First, it is the ability of a component to be used in multiple configurations. Second, it is a necessary factor concerning future requirements and enhancements. 


\section{F-2 Maintainability}

In (IEEE, 2010), maintainability is defined as "the ease with which a software system or component can be modified to change or add capabilities, correct faults or defects, improve performance or other attributes, or adapt to a changed environment". Two metrics, $\mathrm{CC}$ and $\mathrm{MI}$, are used to measure maintainability.

Maintainability is related to reuse in terms of error tracking and debugging. If a component is maintainable it is more likely to be reused. In cases where OSS components are running on systems connected to another system then a bug is particularly problematic. Sometimes debugging a component on one configuration may not work on other configurations. On the other hand, in black box reuse, maintainability is not considered a factor of reusability.

\section{F-3 Portability}

It is defined as "the ease with which a system or component can be transferred from one hardware or software environment to another". The portability of a component depends on its independence, i.e. the ability of the component to perform its functionality without external support. In a scenario where an open source component is used in SPL development, the component should have the characteristic of portability. The component, being a core asset, may be used in the development of another product/family member within the product line/family.

Portability is considered a factor in the sense that a cohesive component is more portable. A component having all the necessary information within it or having less interaction with another module during its execution is more reusable. Again, in the case of black box, portability is not a factor.

\section{F-4 Scope Coverage}

It is the factor that counts the number of features provided by the component from the list of features in the SPL scope.

Another characteristic of the open source components explored is the extent of their scope. A developer would prefer a component to cover as much of the application's functionality as possible. Size is a concern in large components as it often means a high level of complexity and poor understandability. Furthermore, scope coverage is important in situations where future enhancements have already been envisioned, or where there is the likelihood that more features will be added in the future.

\section{F-5 Stability}

Stability of a component refers to its quality of being error free. Here, the term 'stability' can be linked to 'safety in numbers', that is, a reasonable number of developers has contributed to the component and, furthermore, a reasonable number have used it. Stability is also related to the usage history of the component. 


\section{F-6 Usage History}

Usage history provides a hint about the usefulness of the component. Another side of usage history is the maturity of the component. The component can be considered mature if it is used in many applications. The use of a component in many applications also reflects its quality of interoperability. It provides confidence to the potential user that the component can be easily adapted. Another aspect of usage history is that the use of a particular OSS in different applications provides an example of usage of a component. This example can be effective for learning purposes.

\section{F-7 Understandability}

It is defined as "the ease with which a system can be comprehended at both the systemorganizational and detailed statement levels" (IEEE, 2010). In (Sant'anna, et al., 2003; Washizaki, Yamamoto, \& Fukazawa, 2003) 'understandability' is considered a factor of reusability.

'Understandability' is related to the maintainability of a component; a component that is easy to understand is easy to maintain. Understandability affects the reliability of a component.

\section{F-8 Variability}

Software variability as defined in (Firesmith, 2003) is the "degree to which something exists in multiple variants, each having the appropriate capabilities". Variability of a software component is related to its reuse as increased variability increases the likelihood of reuse. The object-oriented variability mechanisms are reported in (Fazal-eAmin, A. K. Mahmood, \& A. Oxley, 2011b). A comprehensive analysis of variability mechanisms is presented in (Fazal-e-Amin, Ahmad Kamil Mahmood, \& Alan Oxley, 2011a).

The two constructs of object oriented paradigm, namely abstract classes and interfaces, support two variability mechanisms, which are 'inheritance' and 'overloading'. These mechanisms facilitates the implementation of positive, negative, optional and alternative types of variability (Fazal-e-Amin, et al., 2011a). Variability can be introduced at class, method and attribute level by using these mechanisms (Fazal-e-Amin, et al., 2011a). (Here the term 'attribute' refers to the data in an objectprogram.) These mechanisms can support open scope variability at compile time (Fazale-Amin, et al., 2011a).

The use of metrics is proposed in this paper as a measure of variability. The abstractness metric computes the ratio of abstract classes and interfaces to the total number of classes. It can be seen as an indirect measure of variability. So, the variability capability of a package, or openness of a package towards variability, can be assessed using the abstractness metric.

Variability affects understandability. Variability is also seen as the configurability of a component, that it can be configured in multiple configurations. Variability is also related to the scalability property of a component, that is, it can be scaled up whenever required. An experiment with human subject is conducted to assess the variability code assets in (Fazal-e-Amin, Ahmad Kamil Mahmood, \& Alan Oxley, 2011c). 


\section{F-9 Documentation}

Documentation affects flexibility, understand-ability and reusability. The issue of documentation is multifaceted. Usually, OSS comes without much documentation. OSS is developed and contributed to by many developers. The number of developers may reach up to thousands, as in case of Linux. The code size increases rapidly. It is very difficult to analyse code without documentation.

Documentation is associated with understand-ability. The lack of documentation, or poorly maintained documentation, hinders understand-ability. Documentation also provides a record of a component; the component history can be known by seeing the documentation.

\section{Metrics}

The following package level metrics are employed in this study. The package level metrics differs from the class level metrics due to the difference in the nature of these artefacts.

\section{Abstractness (A)}

The abstractness of a package is the ratio of the number of abstract classes and interfaces to the total number of classes (Robert Cecil Martin \& Martin, 2006). Its domain is the set of real numbers $[0,1]$, where zero refers to a concrete package, i.e. the absence of an abstract class or interface, and 1 refers to an abstract package, i.e. where all of its classes are abstract.

$A=$ Number of abstract classes \& interfaces / Total number of classes

\section{Cyclomatic Complexity $(C C)$}

The cyclomatic complexity metric a measure of control structure complexity (Lincke \& Lowe, 2007). It counts the linear independent paths, i.e. the minimum number of parts during the execution. It is measured on an absolute scale and its domain is the set of integers $[1, \infty)$.

\section{Fan-in / Afferent Coupling}

The metric 'fan-in' is related to the total number of external classes coupled to the classes of the package. It counts the number of classes outside the package that refer to a class of the given package (Lincke \& Lowe, 2007). Each class is counted for one time only. The value of the metric is zero if there is no external package that refers to the classes of the given package. This metric is equivalent to afferent coupling. It is measured on an absolute scale; its domain is the set of integers $[0, \infty)$. A large value of fan-in represents a high dependability of other packages on the given package.

\section{Fan-out / Efferent Coupling}

The metric 'fan-out' is related to the total number of external classes coupled to the classes of the package. It counts the number of classes outside the package referenced by a class of the given package (Lincke \& Lowe, 2007). Fan-out is equivalent to 
efferent coupling. Each class is counted for one time only. The value of the metric is zero if the package is not referring to any class of an external package. It is measured on an absolute scale; its domain is the set of integers $[0, \infty)$. A large value of fan-out represents a high dependability of the package on other packages.

\section{Instability (I)}

The instability of a package is related to the total number of external classes coupled to the classes of the package. (Robert C. Martin, 2009; Robert Cecil Martin \& Martin, 2006). The instability of a package is the ratio of efferent coupling (fan-out) to the total coupling (fan-out + fan-in). It is represented by ' $I$ ' and its domain is the set of integers $[0,1)$.

$I=C e /(C a+C e) \quad($ eq. 1$)$

where $C e$ is efferent coupling

$\mathrm{Ca}$ is afferent coupling

The instability metric is also an indicator of the resilience to change. The value of 0 represents a stable package, i.e. a package that is little affected by change. The value of 1 represents an unstable package, i.e. a package that is highly affected by change.

\section{Lines of Code (LOC)}

This is a measure of the lines of source code (Fenton \& Pfleeger, 1997). It indicates the size of the entity. The size of the software affects its understandability. It is measured on an absolute scale; its domain is the set of integers $[0, \infty)$.

\section{Lines of Comments}

It is a measure of total number of comment lines in the package, measured on an absolute scale and its domain is the set of integers $[0, \infty)$. 'Comments' have a positive effect on the understandability of a code asset.

\section{Number of Classes or Interfaces}

It is a measure of total number of classes in a package. The size of package affects the understandability. The number of classes is measured on an absolute scale; its domain is the set of integers $[0, \infty)$.

\section{Number of Methods (NOM)}

This metric is introduced in (Li \& Henry, 1993). It measures the number of methods declared within the class. It is an indicator of the size of a class. It is measured on an absolute scale; its domain is the set of integers $[0, \infty)$. 


\section{Maintainability Index}

Maintainability index (MI) (Coleman, Lowther, \& Oman, 1994) value is the representative of the relative maintainability of the code (Laired \& Brennan, 2006). Maintainability index is calculated by making use of lines of code, CCabe complexity metric and Halstead measures. The maintainability index is calculated by following formula:

$\mathrm{MI}=171-5.2 \ln (\mathrm{a} V)-0.23 \mathrm{a} V\left(g^{\prime}\right)-16.2 \ln (\mathrm{aLOC})+50 \sin \left[(2.4 * \text { perCM })^{1 / 2}\right]$ (eq. 2$)$

Where

$\mathrm{a} V=$ average Halstead volume per module

$\mathrm{a} V\left(g^{\prime}\right)=$ average extended cyclomatic complexity per module

aLOC $=$ average count of lines of code per module

perCM $=$ average percent of lines of comments per module

\section{Application of Metrics}

In this section the results of an experiment are presented. This experiment is intended to test the hypotheses formulated as a result of the interviews and survey. In this experiment the hypotheses related to a 'package level reusability attribute model' are tested. (The term 'reusability attribute model' is used for consistency with our earlier work. In the context of this paper it is preferable to think of it as a reusability factor model.) The values of reusability are calculated using the equations stated later in this paper. Pearson's correlation analysis is conducted using statistical software. The results are presented in the form of scatter plots along with the correlation values between 'reusability' and its factors.

Table 3. Attribute, Sub-attribute and Metrics

\begin{tabular}{|l|l|l|}
\hline Attribute & Sub-attribute & Metrics \\
\hline Flexibility & Instability & $\begin{array}{l}\text { I (Robert C. Martin, 2009; Robert } \\
\text { Cecil Martin \& Martin, 2006) }\end{array}$ \\
\hline Understand ability & Comments, Size & $\begin{array}{l}\text { Number of classes, \%comments, } \\
\text { LOC (Fenton \& Pfleeger, 1997), } \\
\text { NOM (Li \& Henry, 1993) }\end{array}$ \\
\hline Portability & Independence & Fan-out (Lincke \& Lowe, 2007) \\
\hline Maintainability & Complexity & $\begin{array}{l}\text { CC (Lincke \& Lowe, 2007), MI } \\
\text { (Coleman, et al., 1994) }\end{array}$ \\
\hline Variability & Abstractness & $\begin{array}{l}\text { A (Robert Cecil Martin \& Martin, } \\
\text { 2006) }\end{array}$ \\
\hline
\end{tabular}


Due to the space limitation detailed explanation of the metrics are provided in this appendices.

Table 4. Pearson's Correlation Values

\begin{tabular}{|c|c|c|c|c|c|c|}
\hline & \multicolumn{6}{|c|}{ Pearson's Correlations } \\
\hline & & Flexibility & $\begin{array}{l}\text { Maintain- } \\
\text { ability }\end{array}$ & Portability & $\begin{array}{l}\text { Understan } \\
\text { d- ability }\end{array}$ & Variability \\
\hline \multirow[t]{3}{*}{ Reusability } & $\begin{array}{l}\text { Pearson } \\
\text { Correlation }\end{array}$ & $.789^{* *}$ & $.667^{* *}$ & $.693^{* *}$ & $.417^{* *}$ & $.674^{* *}$ \\
\hline & Sig. (2-tailed) & .000 & .000 & .000 & .000 & .000 \\
\hline & $\mathrm{N}$ & 77 & 77 & 77 & 77 & 77 \\
\hline & \multicolumn{6}{|c|}{ **. Correlation is significant at the 0.01 level (2-tailed). } \\
\hline
\end{tabular}

\section{DISCUSSIONS}

At the outset of this research nine factors were identified as affecting reusability. We will now discuss, in turn, each of these factors. The first factor identified as affecting reusability is flexibility of the software. The survey results show a strong indication that 'flexibility' is one of the factors of reusability. The metrics shows that the strength of correlation between flexibility and reusability is .789 .

The second factor identified as affecting reusability is maintainability of the software. The survey results show a strong indication that 'maintainability' is a factor of reusability. The metrics show that the strength of correlation between maintainability and reusability is .667 .

The third factor identified as affecting reusability is portability of the software. The survey results show that the population has little confidence that 'portability' is one of the factors of reusability. The metrics show that the strength of correlation between portability and reusability is .693 .

The fourth factor identified as affecting reusability is scope coverage of the software. The survey results show that there is some doubt as to whether or not 'scope coverage' is a factor of reusability.

The fifth factor identified as affecting reusability is stability of the software. The survey results point towards the importance of 'stability' as a factor of reusability in the scenario studies in this research.

The sixth factor identified as affecting reusability is understandability of the software. The survey results show that there is a strong indication that 'understandability' is a factor of reusability. The metrics show that the strength of correlation between understandability and reusability is .417 . 
The seventh factor identified as affecting reusability is the usage history of the software. The above mentioned empirical evidence points towards the importance of 'usage history' as a factor of reusability in the scenario studies in this research.

The eighth factor identified as affecting reusability is variability of the software. The survey results show that there is a consensus that 'variability' is a factor of reusability. The metrics show that the strength of correlation between variability and reusability is .674 .

The ninth factor identified as affecting reusability is documentation of the software. The survey results show that there is a strong indication that 'documentation' is a factor of reusability.

\section{Threats to Validity of Results}

The validity of quantitative results is affected by type I and type II errors. Both of these error types are related to the rejection or acceptance of the null hypothesis. In this research study, a low value of probability is used, i.e. 0.01. This low level of the p-value ensures that there is no type I or type II errors in the results.

The validity of qualitative research is of four types: descriptive, interpretive, concurrent and theoretical. Descriptive validity is related to the reporting of events, behaviors, settings, people, places and times (Sullivan, 2009). It is of little concern to this study. Interpretive validity (Johnson \& Christensen, 2011) is more of a concern to this research. Whenever there is ambiguity, the transcriptions are reviewed by the researcher to ensure the interpretive validity of the results. Furthermore, the findings of the qualitative studies are provided to the respondents. This measure was taken to cater for the possible apprehensions of respondents about the results. The respondents verified the interpretations.

Theoretical validity (Sullivan, 2009) is maintained by comparing the findings of this research study with contemporary studies. It can be safely said that the findings presented in this study are in line with the available theory.

Concurrent validity (Basit, 2010) is exhibited by the fact that the qualitative data is collected using five interviews. Similar patterns and trends are identified from the collected data. The only findings that are reported are those which are concurrent, i.e. extracted from multiple respondents.

\section{Limitations}

The approach presented in this paper is meant to be of use to software engineers etc. The approach is applied on open source software to obtain the results. The findings are specific to open source projects. The results are acquired by analysing the source code. Therefore, the results may not comply with black-box reuse, i.e. in which the user has no access to the source code of the project.

The metrics used to assess the factors are generic object oriented metrics. However, the data set used in the statistical experiment consists of projects implemented in Java. Therefore, any results obtained from using some other programming language may differ from the results of this study. 


\section{CONCLUSIONS}

In this study, the factors affecting reusability of OSS in a reuse-intensive software development environment have been identified. These factors include the following: flexibility, understand-ability, maintainability, portability, scope-coverage, stability, usage history, variability and documentation. A survey was conducted to ascertain the relative rankings of these factors. The survey results show a high ranking for understand-ability, flexibility, maintainability, documentation and usage history. The statistical results of an experiment show a strong correlation between four of the five factors, 'understand-ability' being the odd one out. Further studies are required in order to identify new metrics that could be used in a similar experiment. The contributions of this study are twofold, first as a demonstration of mixed method research in software engineering. Second, the topic of reusability is revisited in the context of OSS and reuse-intensive software development.

\section{REFERENCES}

Ågerfalk, P., Fitzgerald, B., Lings, B., Lundell, B., O’Brien, L., \& Thiel, S. (2006). Open Source in Software Product Line: An Inevitable Trajectory. Paper presented at the 10th International Software Product Line Conference (SPLC '06).

Ågerfalk, P. J., Deverell, A., Fitzgerald, B., \& Morgan, L. (2005). Assessing the Role of Open Source Software in the European Secondary Software Sector: A Voice from Industry. Paper presented at the First International Conference on Open Source Systems, Genova.

Ahmed, F., Capretz, L. F., \& Babar, M. A. (2008, July 28 2008-Aug. 1 2008). A Model of Open Source Software-Based Product Line Development. Paper presented at the Computer Software and Applications, 2008. COMPSAC '08. 32nd Annual IEEE International.

Basit, T. N. (2010). Conducting Resaerch in Educational Contexts Continuum International Publishing Group.

Beck, K. (2011). Confidence Intervals, 2011, from http://www.doh.wa.gov/healthyyouth/technical/confidinterval.htm

Boxall, M. A. S., \& Araban, S. (2004). Interface Metrics for Reusability Analysis of Components. Paper presented at the Proceedings of the 2004 Australian Software Engineering Conference.

Burgin, M., Lee, H. K., \& Debnath, N. (2004, 8-10 Nov. 2004). Software technological roles, usability, and reusability. Paper presented at the Proceedings of the 2004 IEEE International Conference on Information Reuse and Integration, IRI 2004.

Coleman, D., Lowther, B., \& Oman, P. (1994). Using Metrics to Evaluate Software System Maintainability Computer, 27(8), 44-49.

Easterbrook, S., Singer, J., Storey, M.-A., \& Damian, D. (2008). Selecting Empirical Methods for Software Engineering Research Guide to Advanced Empirical Software Engineering (pp. 285-311).

Elo, S., \& Kyngäs, H. (2008). The qualitative content analysis process. Journal of Advanced Nursing, 62(1), 107-115. doi: 10.1111/j.1365-2648.2007.04569.x 
Fazal-e-Amin, Mahmood, A. K., \& Oxley, A. (2011a). An analysis of object oriented variability implementation mechanisms. SIGSOFT Softw. Eng. Notes, 36(1), 1-4. doi: $10.1145 / 1921532.1921538$

Fazal-e-Amin, Mahmood, A. K., \& Oxley, A. (2011b). Mechanisms for managing variability when implementing object oriented components. Paper presented at the National Information Technology Symposium (NITS), King Saud University, KSA.

Fazal-e-Amin, Mahmood, A. K., \& Oxley, A. (2011c). Metrics Based Variability Assessment of Code Assets In J. M. Zain, W. M. b. Wan Mohd \& E. ElQawasmeh (Eds.), Software Engineering and Computer Systems (Vol. 181, pp. 66-75): Springer Berlin Heidelberg.

Fazal-e-Amin, Mahmood, A. K., \& Oxley, A. (2011d). A Review of Software Component Reusability Assessment Approaches. Research Journal of Information Technology, 3(1), 1-10.

Fazal-e-Amin, Mahmood, A. K., \& Oxley, A. (2011e, 25-28 Sept. 2011). Using open source components in software product lines - An exploratory study. Paper presented at the IEEE Conference on Open Systems (ICOS) 2011.

Fenton, N., \& Pfleeger, S. (1997). Software Metrics: A Rigorous and Practical Approach (2nd ed.): PWS Publishing Co.

Firesmith, D. (2003). Common Concepts Underlying Safety, Security, and Survivability Engineering. Pittsburgh, PA, USA: Software Engineering Institute, Carnegie Mellon University.

Frakes, W. B., \& Kyo, K. (2005). Software reuse research: status and future. IEEE Transactions on Software Engineering, 31(7), 529-536.

Gravetter, F. J., \& Forzano, L. A. B. (2011). Research Methods for the Behavioral Sciences (4th ed.): Cengage Learning.

Gray, D. E. (2009). Doing Research in the Real World (2nd ed.): SAGE Publication Ltd.

Gui, G., \& Scott, P. D. (2007). Ranking reusability of software components using coupling metrics. Journal of Systems and Software, 80(9), 1450-1459. doi: http://dx.doi.org/10.1016/j.jss.2006.09.048

Gui, G., \& Scott, P. D. (2008, 18-21 Nov. 2008). New Coupling and Cohesion Metrics for Evaluation of Software Component Reusability. Paper presented at the The 9th International Conference for Young Computer Scientists, 2008. ICYCS 2008. .

Harman, M. (2010, 12-13 Sept. 2010). Why Source Code Analysis and Manipulation Will Always be Important. Paper presented at the Source Code Analysis and Manipulation (SCAM), 2010 10th IEEE Working Conference on.

Höst, M., Oručević-Alagić, A., \& Runeson, P. (2011). Usage of Open Source in Commercial Software Product Development - Findings from a Focus Group Meeting. In D. Caivano, M. Oivo, M. Baldassarre \& G. Visaggio (Eds.), ProductFocused Software Process Improvement (Vol. 6759, pp. 143-155): Springer Berlin / Heidelberg.

Howe, C. (2000). Open Source Cracks The Code: Forrester Research.

Hsieh, H.-F., \& Shannon, S. E. (2005). Three Approaches to Qualitative Content Analysis. Qualitative Health Research, 15(9), 1277-1288.

Hummel, O., Janjic, W., \& Atkinson, C. (2008). Code Conjurer: Pulling Reusable Software out of Thin Air. IEEE Software, 25(5), 45-52. 
IEEE. (2010). Systems and Software Engineering Vocabulary ISO/IEC/IEEE 24765:2010 (pp. 1-418).

Johnson, B., \& Christensen, L. (2011). Educational Resaerch : Quantitative, Qualitative, and Mixed Approaches (4th ed.): Sage Publication, Inc.

Kenwood, C. A. (2001). A Business Case Study of Open Source Software: The MITRE Corporation.

Krippendorff, K. (2004). Content analysis: an introduction to its methodology (2nd ed.): Sage.

Krishnamurthy, S. (2003). A Managerial Overview of Open Source Software. Business Horizons, September-October 2003.

Laired, L. M., \& Brennan, M. C. (2006). Software Measurement and Estimation : A Practical Approach: John Wiley \& Sons, Inc.

Lazaro, M., \& Marcos, E. (2006). An Approach to the Integration of Qualitative and Quantitative Research Methods in Software Engineering Research. Paper presented at the 2nd International Workshop on Philosophical Foundations of Information Systems Engineering (PHISE'06).

Leech, N., \& Onwuegbuzie, A. (2009). A typology of mixed methods research designs. Quality \& Quantity, 43(2), 265-275. doi: 10.1007/s11135-007-9105-3

Li, W., \& Henry, S. (1993, 21-22 May 1993). Maintenance metrics for the object oriented paradigm. Paper presented at the Proceedings of First International Software Metrics Symposium, 1993.

Lincke, R., \& Lowe, W. (2007). Compendium of Software Quality Standards and Metrics - Version 1.0.

Linden, F., Schmid, K., \& Rommes, E. (2007). Software Product Lines in Action: The Best Industrial Practice in Product Line Engineering: Springer-Verlag Berlin Heidelberg.

Linden, F. v. d., Lundell, B., \& Marttiin, P. (2009). Commodification of Industrial Software: A Case for Open Source. IEEE Software, 26(4), 77-83. doi: $10.1109 / \mathrm{ms} .2009 .88$

Martin, R. C. (2009). Clean Code: Pearson Education, Inc.

Martin, R. C., \& Martin, M. (2006). Agile Principles, Patterns, and Practices in C\#: Prentice Hall.

Niemi, T., Tuisku, M., Hameri, A.-p., \& Curtin, T. (2009). Server-Based Computing Solution Based on Open Source Software. Information Systems Management, 26(1), 77-86. doi: 10.1080/10580530802552227

Pohl, K., Böckle, G., \& Linden, F. v. d. (2005). Software Product Line Engineering Foundations, Principles, and Techniques: Springer-Verlag Berlin Heidelberg.

Punch, K. F. (2009). Introduction to Research Methods in Education Sage Publications Ltd.

Samadi, S., Almaeh, N., Wolfe, R., Olding, S., \& Isaac, D. (2004, 20-24 Sept. 2004). Strategies for enabling software reuse within the Earth Science Community. Paper presented at the Proceedings of IEEE International Geoscience and Remote Sensing Symposium, 2004. IGARSS '04. .

Sant'anna, C., Garcia, A., Chavez, C., Lucena, C., \& von Staa, A. (2003). On the Reuse and Maintenance of Aspect-Oriented Software: An Assessment Framework. Paper presented at the Proceedings XVII Brazilian Symposium on Software Engineering. 
Sharma, A., Grover, P. S., \& Kumar, R. (2009). Reusability assessment for software components. SIGSOFT Softw. Eng. Notes, 34(2), 1-6. doi: http://doi.acm.org/10.1145/1507195.1507215

Sommerville, I. (2007). Software Engineering (8th ed.): Addison-Wesley.

Stafford, J. (2006). Time to plan your company's escape from Microsoft. SearchExchange.com Retrieved 01 June 2011, from http://searchenterpriselinux.techtarget.com/news/1163576/2006-Time-to-planyour-companys-escape-from-Microsoft

Sullivan, L. E. (2009). The SAGE Glossary of the Social and Behavioral Sciences: Sage Publications, Inc.

Tashakkori, A., \& Teddlie, C. (1998). Mixed Methodology Combining Qualitative and Quantitative Approaches: SAGE Publications, Inc.

Washizaki, H., Yamamoto, H., \& Fukazawa, Y. (2003). A Metrics Suite for Measuring Reusability of Software Components. Paper presented at the Proceedings of the 9th International Symposium on Software Metrics.

Wasserman, A. (2011). How the Internet transformed the software industry. Journal of Internet Services and Applications, 2(1), 11-22. doi: 10.1007/s13174-011-0019-x

Wheeler, D. A. (2005). Why Open Source Software / Free Software (OSS/FS, FLOSS, or FOSS)? Look at the Numbers! Retrieved 01 June, 2011, from http://www.dwheeler.com/oss_fs_why.html

Wood, M., Daly, J., Miller, J., \& Roper, M. (1999). Multi-method research: An empirical investigation of object-oriented technology. Journal of Systems and Software, 48(1), 13-26.

Yoonjung, C., Sungwook, L., Houp, S., Jingoo, P., \& SunHee, K. (2008, 17-20 Feb. 2008). Practical S/W Component Quality Evaluation Model. Paper presented at the 10th International Conference on Advanced Communication Technology, 2008. ICACT 2008. . 